\title{
Antibiotic sensitivity of common respiratory bacteria of pig from Hubei province, China
}

\author{
Anh L. L. Nguyen ${ }^{1 *}$, An T. T. Vo ${ }^{1}$, \& Qigai $\mathrm{He}^{2}$ \\ ${ }^{1}$ Faculty of Animal Science and Veterinary Medicine, Nong Lam University, Ho Chi Minh City, Vietnam \\ ${ }^{2}$ Huazhong Agricultural University, Wuhan, China
}

\author{
ARTICLE INFO \\ Research Paper \\ Received: February 27, 2020 \\ Revised: April 17, 2020 \\ Accepted: May 19, 2020 \\ Keywords \\ Antibiotic resistance \\ Pigs \\ Respiratory bacteria \\ * Corresponding author \\ Nguyen Luong Lam Anh \\ Email: lamanhft@gmail.com
}

\begin{abstract}
The use of antimicrobials for feeding and treatment is crucial to animal health. However, continuous use of antibiotics is contributing to emergence and widespread of antibiotic resistance. This study aimed to investigate the antimicrobial resistance of five major respiratory pathogens in pigs of Hubei province, China, from October to December, 2019. Antibiotic susceptibility testing for Streptococcus suis, Haemophilus parasuis, Pasteurella multocida, Bordetella bronchiseptica and Actinobacillus pleuropneumoniae was determined to representatives of relevant antibiotic classes.
\end{abstract}

Streptococcus suis isolates were mostly sensitive to beta-lactams, whereas high levels of resistance were observed to quinolones, gentamycin, doxycycline, trimethoprime and lincomycin. For H. parasuis, P. multocida and A. pleuropneumoniae of Pasteurellaceae family, the susceptibility to beta-lactams and quinolones was displayed. Most B. bronchiseptica isolates were sensitive to doxycycline, azithomycin, polymycin whereas high resistance levels to betalactams, aminoglycosides and quinolones were recorded.

This study obtained practical data for later studies and usage to combat infections due to respiratory bacteria.

Cited as: Nguyen, A. L. L., Vo, A. T. T., \& He, Q. (2020). Antibiotic sensitivity of common respiratory bacteria of pig from Hubei province, China. The Journal of Agriculture and Development 19(3), 16-21.

\section{Introduction}

Porcine respiratory diseases complex is caused by multifactorial aetiologies, including the viral and bacterial pathogens, the environment, management and genetic factors. Within this complex, Streptococcus suis, Haemophillus parasuis, Pasteurella multocida, Actinobacillus pleuropneumoniae and Bordetella bronchiseptica have been known to be ubiquitous in almost all pig farms. S. suis is as a major respiratory commensal and pathogen of pigs and an emerging zoonotic agent of meningitis in human (GoyetteDesjardins et al., 2014). Haemophillus parasuis produces Glässer's disease as well as pneumonia (Nedbalcova et al., 2006). Pasteurella mul- tocida causes atrophic rhinitis, particularly when combined with B. bronchiseptica (Jeffrey et al., 2013). Actinobacillus pleuropneumoniae generates contagious hemorrhagic pleuropneumonia in pigs (Brownfield, 2013). Due to their complexity and indeterminacy, bacterial diseases are very challenging to control.

Antimicrobial agents are important for effective production of food animals as growth promoter or/and disease prevention. As the world's largest pork producer and consumer, China has been reported for the massive use of antibiotic in food animal production. Zhao et al. (2011) showed antimicrobial susceptibility tests on $B$. bronchiseptica isolates from Chinese farms that were highly resistant to ampicillin, cefazolin, 
streptomycin, amoxicillin and tetracycline. Zhang et al. (2015) found the most antibiotics consumed in China's swine farming were fluoroquinolones and $\beta$-lactams. Therefore, antimicrobial surveillance is necessary to provide a better understanding of antibiotic resistance in the animal population.

This study aimed to contribute the comprehension of the antibiotic susceptibility pattern of $S$. suis, H. parasuis, P. multocida, A. pleuroplneumoniae and B. bronchiseptica, the five important pathogens found in the respiratory tract of pigs in Hubei province, China, using disk diffusion test.

\section{Materials and Methods}

\subsection{Sample collection}

From October to December 2019, a total of 155 samples from 14 different pig farms in Hubei province were sent to the Animal Diagnostic Center of Huazhong University. The collected samples included lungs, spleen, synovial fluid, brain, tracheal effusion etc. Lived pigs were observed for evaluating clinical signs and endured necropsy to collect samples. For every individual pig, lung and spleen samples were sealed in a clean zipper bag; brain and synovial fluid were kept in an eppendorf tubes (EP tube). Nasal samples were collected by using sterile cotton swabs and placed in sterilized EP tubes. The samples were clearly marked.

After the period of three months, 133 strains of the five concerned bacteria species from 155 samples were isolated and identified by using multiplex PCR assays. For the identification of the five bacteria, the primers of following target genes were used: $16 \mathrm{~S}$ rRNA to detect $S$. suis (Cheung, 2008), 16S rRNA for H. parasuis, apxIV for A. pleuropneumoniae, fla for B. bronchiseptica (Xue, 2009) and ktm1 for P. multocida (Nagai et al., 1994). The greatest number of isolated strains were obtained from $S$. suis $(40 \%, 62 / 155)$, followed by H. parasuis $(18.71 \%$, 29/155), P. multocida (14.83\%, 23/155), B. bronchiseptica $(8.39 \%, 13 / 155)$, and A. pleuropneumoniae $(3.87 \%, 6 / 155)$.

\subsection{Kirby-Bauer antibiotic testing}

Twenty antibiotic agents (Hangzhou Binhe Microorganism Reagent Co., Ltd) were used, including cefotazime $(30 \mu \mathrm{g})$, cephradine $(30 \mu \mathrm{g})$, ceftriaxone $(30 \mu \mathrm{g})$, ceftazidime $(30 \mu \mathrm{g})$, amox- icillin $(20 \mu \mathrm{g})$ and ampicillin $(10 \mu \mathrm{g})$, ofloxacin (5 $\mu \mathrm{g})$, ciprofloxacin $(5 \mu \mathrm{g})$, enrofloxacin (10 $\mu \mathrm{g})$, norfloxacin (10 $\mu \mathrm{g})$, spectinomycin (100 $\mu \mathrm{g})$, gentamicin $(10 \mu \mathrm{g})$, streptomycin $(10 \mu \mathrm{g})$, amikacin $(30 \mu \mathrm{g})$, kanamycin $(30 \mu \mathrm{g})$, doxycycline $(30 \mu \mathrm{g})$, lincomycin $(30 \mu \mathrm{g})$, azithromycin $(15 \mu \mathrm{g})$, polymyxin B (300 $\mu \mathrm{g})$ and trimethoprim $(23.75 / 1.25 \mu \mathrm{g})$.

Each purified isolates of tested bacteria were evenly spread onto a tryptic soy agar plate (TSA, BD ${ }^{\mathrm{TM}}$, USA) that had been coated with nicotinamide adenine dinucleotide liquid (NAD, Guangzhou Saiguo Biotech, China) and bovine serum (Zhejiang Tianhang Biotechnology, China). The antimicrobial discs were placed onto the surface of the agar. The plates were then incubated at $37^{\circ} \mathrm{C}$ for about $24 \mathrm{~h}$. The inhibition zone diameter was measured and compared with standardized CLSI interpretive criteria to designate the isolate as sensitive, intermediate or resistant to the drug (CLSI, 2018). In this study, the isolates that showed intermediate were classified as resistant.

\subsection{Results and Discussion}

The resistant and sensitive rates of the five bacteria species to 20 antibiotic agents are presented in Table 1. Results showed the resistance rates of $S$. suis strains to quinolones, aminoglycosides, macrolides, lincomycins, tetracyclines, polymyxins and sulfonamides were all over $60 \%$. $H$. pasasuis strains were sensitive to majority of the drugs but highly resistant to amoxicillin, streptomycin, amikacin, kanamycin and lincomycin. The resistance of $P$. multocida strains to aminoglycosides and lincosamides were apparently high compared to other antibiotic groups (Table 1).

With the small number of isolates being tested, the two purified isolates of A. pleuropneumoniae were sensitive to beta-lactams, quinolones and aminoglycosides. In contrast, all of the three isolates of $B$. bronchiseptica resisted to those drugs and only sensed to doxycycline, gentamicin, azithromycin and polymyxin B.

The drug-resistance pattern of bacterial isolates obtained in this study indicates that $S$. suis, H. parasuis, P. multocida, B. bronchiseptica and A. pleuropneumoniae displayed high antibiotic resistance rates to 8 tested antibiotics/antimicrobial classes. The resistance proportion of $S$. suis to these antibiotics were all 
Table 1. Antibiotic susceptibility rates (\%) and number of S. suis, H. parasuis and $P$. multocida isolates (in brackets) from infected pigs of Hubei province

\begin{tabular}{|c|c|c|c|c|c|c|}
\hline \multirow{2}{*}{ Antibiotics } & \multicolumn{2}{|c|}{ S. suis } & \multicolumn{2}{|c|}{ H. pasasuis } & \multicolumn{2}{|c|}{ P. multocida } \\
\hline & Sensitive & Resistant & Sensitive & Resistant & Sensitive & Resistant \\
\hline \multirow{2}{*}{ Amoxicillin } & 62.5 & 37.5 & 20.0 & 80.0 & 25.0 & 75.0 \\
\hline & $(5)$ & $(3)$ & $(1)$ & $(4)$ & $(1)$ & $(3)$ \\
\hline \multirow{2}{*}{ Ampicillin } & 75.0 & 25.0 & 50.0 & 50.0 & 40.0 & 60.0 \\
\hline & $(6)$ & $(2)$ & $(2)$ & $(2)$ & $(2)$ & $(3)$ \\
\hline \multirow{2}{*}{ Ceftiaxone } & 75.0 & 25.0 & 80.0 & 20.0 & 60.0 & 40.0 \\
\hline & $(6)$ & $(2)$ & $(4)$ & $(1)$ & $(3)$ & $(2)$ \\
\hline \multirow{2}{*}{ Cefotaxime } & 100.0 & 0.0 & 75.0 & 25.0 & 50.0 & 50.0 \\
\hline & $(6)$ & $(0)$ & $(3)$ & $(1)$ & $(2)$ & $(2)$ \\
\hline \multirow{2}{*}{ Ceftazidime } & 25.0 & 75.0 & 66.7 & 33.3 & 0.0 & 100.0 \\
\hline & $(1)$ & $(3)$ & $(2)$ & $(1)$ & $(0)$ & $(1)$ \\
\hline \multirow{2}{*}{ Cefradine } & 80.0 & 20.0 & 60.0 & 40.0 & 100.0 & 0.0 \\
\hline & $(4)$ & $(1)$ & $(3)$ & $(2)$ & $(4)$ & $(0)$ \\
\hline \multirow{2}{*}{ Ofloxacin } & 25.0 & 75.0 & 80.0 & 20.0 & 80.0 & 20.0 \\
\hline & $(2)$ & $(6)$ & $(4)$ & $(1)$ & $(4)$ & $(1)$ \\
\hline \multirow{2}{*}{ Ciprofloxacin } & 0.0 & 100.0 & 50.0 & 50.0 & 80.0 & 20.0 \\
\hline & $(0)$ & $(8)$ & $(2)$ & $(2)$ & $(4)$ & $(1)$ \\
\hline \multirow{2}{*}{ Enrofloxacin } & 50.0 & 50.0 & 75.0 & 25.0 & 100.0 & 0.0 \\
\hline & $(4)$ & $(4)$ & $(3)$ & $(1)$ & $(4)$ & $(0)$ \\
\hline \multirow{2}{*}{ Norfloxacin } & 0.0 & 100.0 & 60.0 & 40.0 & 60.0 & 40.0 \\
\hline & $(0)$ & $(8)$ & $(3)$ & $(2)$ & $(3)$ & $(2)$ \\
\hline \multirow{2}{*}{ Spectinomycin } & 37.5 & 62.5 & 80.0 & 20.0 & 40.0 & 60.0 \\
\hline & $(3)$ & $(5)$ & $(4)$ & $(1)$ & $(2)$ & $(3)$ \\
\hline \multirow{2}{*}{ Gentamicin } & 0.0 & 100.0 & 40.0 & 60.0 & 20.0 & 80.0 \\
\hline & $(0)$ & $(8)$ & $(2)$ & $(3)$ & $(1)$ & $(4)$ \\
\hline \multirow{2}{*}{ Streptomycin } & 0.0 & 100.0 & 0.0 & 100.0 & 0.0 & 100.0 \\
\hline & $(0)$ & $(6)$ & $(0)$ & $(1)$ & $(0)$ & $(2)$ \\
\hline \multirow{2}{*}{ Amikacin } & 0.0 & 100.0 & 0.0 & 100.0 & 20.0 & 80.0 \\
\hline & $(0)$ & $(8)$ & $(0)$ & $(5)$ & $(1)$ & $(4)$ \\
\hline \multirow{2}{*}{ Kanamycin } & 0.0 & 100.0 & 0.0 & 100.0 & 20.0 & 80.0 \\
\hline & $(0)$ & $(7)$ & $(0)$ & $(5)$ & $(1)$ & $(4)$ \\
\hline \multirow{2}{*}{ Doxycycline } & 25.0 & 75.0 & 100.0 & 0.0 & 60.0 & 40.0 \\
\hline & $(2)$ & $(6)$ & $(5)$ & $(0)$ & $(3)$ & $(2)$ \\
\hline \multirow{2}{*}{ Lincomycin } & 0.0 & 100.0 & 0.0 & 100.0 & 0.0 & 100.0 \\
\hline & $(0)$ & $(8)$ & $(0)$ & $(5)$ & (0) & $(5)$ \\
\hline \multirow{2}{*}{ Azithromycin } & 12.5 & 87.5 & 60.0 & 40.0 & 60.0 & 40.0 \\
\hline & $(1)$ & $(7)$ & $(3)$ & $(2)$ & $(3)$ & $(2)$ \\
\hline \multirow{2}{*}{ Polymyxin B } & 0.0 & 100.0 & 60.0 & 40.0 & 60.0 & 40.0 \\
\hline & $(0)$ & $(8)$ & $(3)$ & $(2)$ & $(3)$ & $(2)$ \\
\hline \multirow{2}{*}{ Trimethoprim } & 12.5 & 87.5 & 60.0 & 40.0 & 60.0 & 40.0 \\
\hline & $(1)$ & $(7)$ & $(3)$ & $(2)$ & $(3)$ & $(2)$ \\
\hline
\end{tabular}

over $60 \%$ except for $\beta$-lactam group. Some antibiotics that used to effectively deal with Gramnegative bacteria (H. parasuis, P. multocida, B. bronchiseptica and $A$. pleuropneumoniae) such as macrolides and beta-lactams were indicated to be less sensitive, especially lincomycin could not be used for any bacterial isolates. Polymycin B, which is known to use in human treatment, presented $100 \%$ resistance by $S$. suis and A. pleuropneumoniae, and $40 \%$ by $H$. parasuis and $P$. multocida. As a result, only a narrow spectrum of effective antibiotic drugs can be used for the treatment of infection in Hubei pigs.

This study also revealed the number of bacte- 
A.

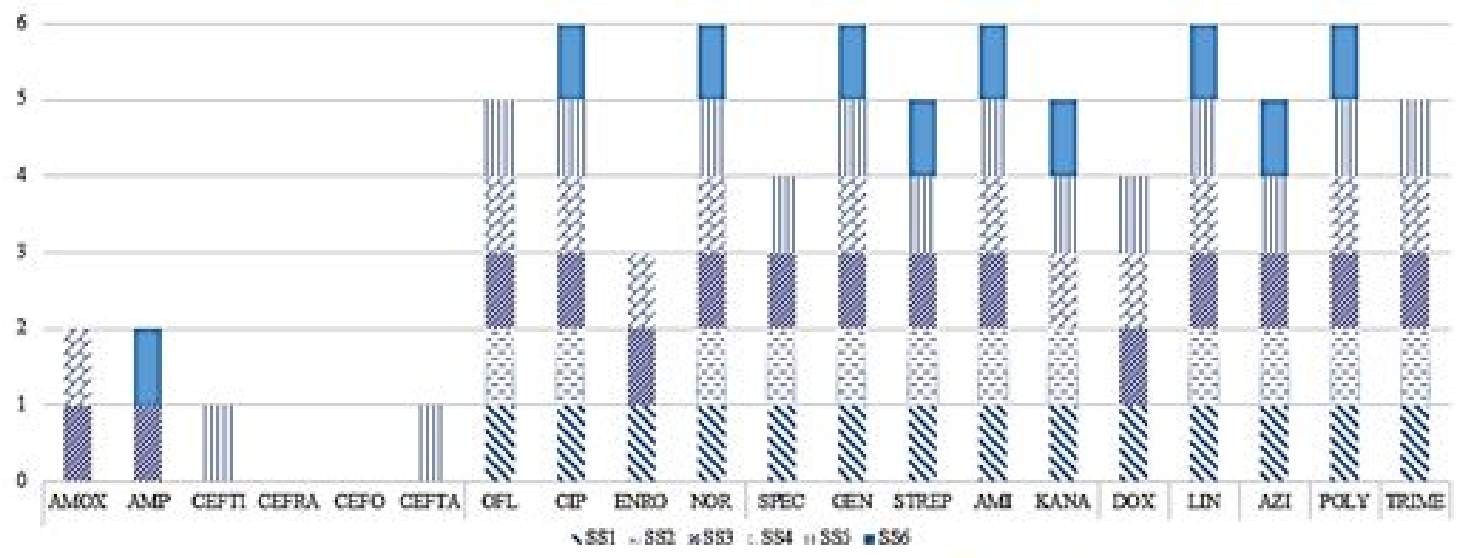

B. 5

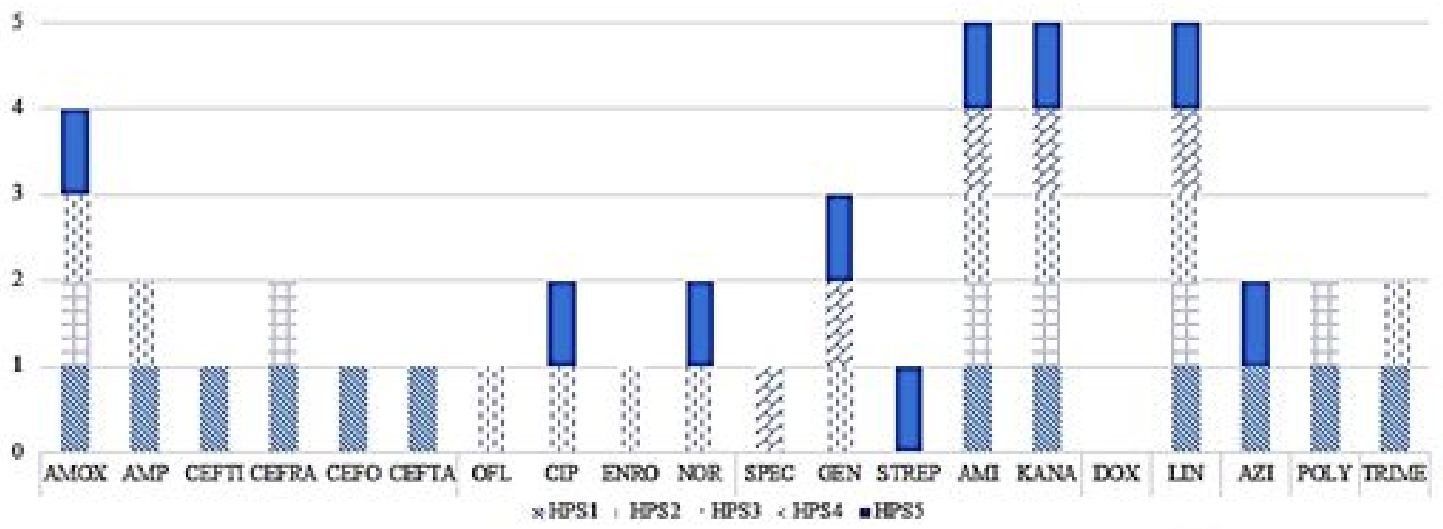

C. 5

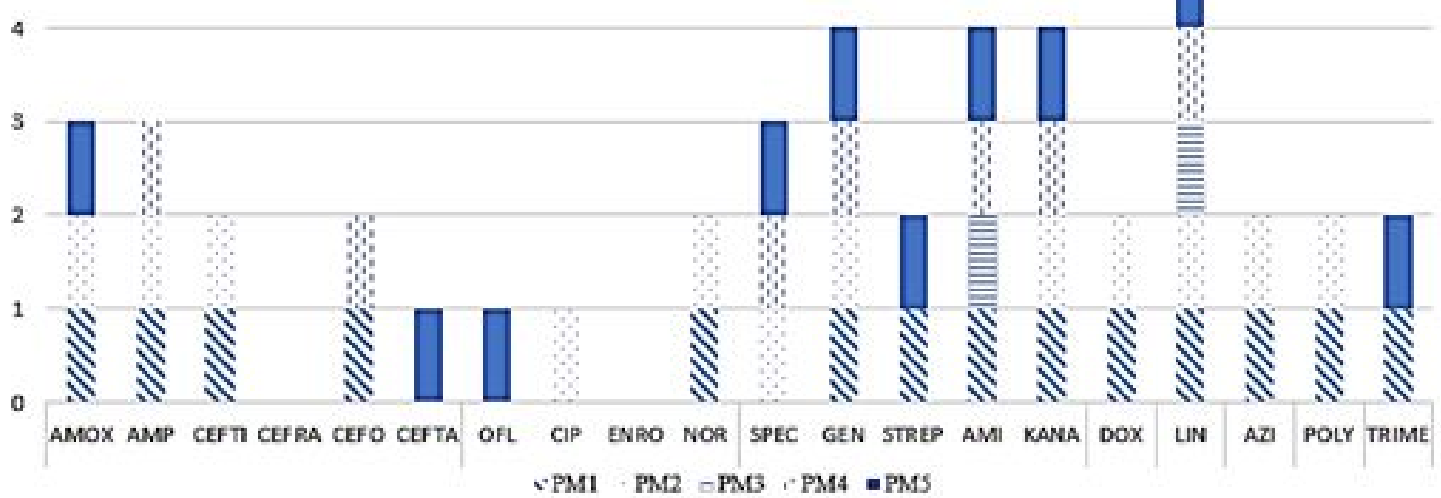

*Note: Beta-lactams (AMOX: amoxicillin, AMP ampicillin, CEFTI: ceftriaxone, CEFRA: cefradine, CEFO: cefotaxime, CEFTA: ceftazidime). Quinolones (OFL: ofloxacin, CIP: ciprofloxacin, ENRO: enrofloxacin, NOR: norfloxacin). Aminoglycosides (SPEC: spectinmycin, GEN: gentamycin, STREP: streptomycin, AMI: amikacin, KANA: kanamycin). Tetracyclines (DOX: doxycycline). Lincosamide (LIN lincomycin). Macrolides (AZI: azithromycin). Polymycin (POLY: polymyxin B). Sulfonamide (TRIME: trimethoprime). SS1 - SS6: S. suis isolates number 1 to 6. HPS1 - HPS6: H. parasuis isolates number 1 to 5. PM1 - PM5: P. multocida isolates number 1 to 5 .

Figure 1. The number of bacterial isolates resistant to antimicrobial agents (A) S. suis isolates.

rial isolates that exhibited multi-drug resistance (MDR) (Figure 1). According to these data, each isolate of $S$. suis were resistant to at least one antimicrobial drug in more than six antimicro- bial categories. Each isolate of $H$. parasuis and $P$. multocida were resistant to at least one antimicrobial drug in two or more antimicrobial categories. The three B. bronchiseptica isolates were 
also against to at least one antimicrobial agent of beta-lactams, quinolones, aminoglycosides and lincosamides. Similarly, A. pleuropneumoniae isolates were resistant to at least one antimicrobial agent of seven tested drug classes, except for macrolides.

The results suggested that five species of bacteria were highly multi-resistant to the eight common drug classes. Multi-drug resistance is a problem that continues to challenge the healthcare sector. Different countries have reported the widespread of clinical resistance due to the massive of antimicrobial drugs (Jong et al. 2018). The transmission of MDR bacteria into the community is seriously associated with increased morbidity, mortality, healthcare costs and antibiotic use. Together with many European countries and the USA, China is preparing a national action to deal with antibiotic resistance. Current technology makes possible the identification of new drugs or inhibitors of resistance mechanisms to extend the life of existing antibiotics, or alternatives like plant extracts (Laxminarayan, 2013). However, these tend to take time and require further efforts. Initial steps to prevent the spreading of MDR is use antibiotics only when needed and correctly, control the usage by reducing antibiotics in livestock management.

Due to different antibiotic usage of different farms, more difficulty and complication have raised in the aspect of antibiotic control of the area. The temporary solution is giving drug regimen based on susceptibility result of individual farms. Long-term plan with a detailed guideline of antibiotic implication should be developed for the control of bacterial disease and protect public health from antimicrobial resistance.

\section{Conclusions}

The results demonstrated high multi-resistance among the five bacterial species to the eight tested antimicrobial classes. The results emphasize the need for continuous surveillance of resistance patterns. Antibiotic prescription guidelines and infection control through the early detection of clinical should be carried out to prevent transmission of pathogens, as well as in the possible incorporation of the prevalent serotypes in the development of new vaccines.

\section{Acknowledgments}

I would like to express my gratitude to Prof. He Qigai and Assoc. Prof. Vo Thi Tra An for being my research supervisors, for their valuable support and advice. I would like to send my special thanks to Dr. Sun Qi for his enthusiastic guidance. This study was supported by the Diagnostic Center for Animal Disease, College of Veterinary Medicine, Huazhong Agricultural University, China. This research was supported by the China Agriculture Research System (No. CARS$35)$.

\section{References}

Brownfield, B. (2013). Actinobacillus pleuropneumoniae in swine. Purdue University-Animal Disease Diagnostic Laboratory. Indiana, USA.

Cheung, P. Y., Lo, K. L., Cheung, T. T., Yeung, W. H., Leung, P. H., \& Kam, K. M. (2008). Streptococcus suis in retail markets: How prevalent is it in raw pork? International Journal of Food Microbiology 127, 316320 .

CLSI (Clinical and Laboratory Standards Institute). (2018). Performance standards for antimicrobial susceptibility testing ( $28^{\text {th }}$ ed.). CLSI document M100S28. Pennsylvania, USA: Clinical and Laboratory Standards Institute.

Goyette-Desjardins, G., Auger, J. P., Xu, J., Segura, M., \& Gottschalk, M. (2014). Streptococcus suis, an important pig pathogen and emerging zoonotic agent-an update on the worldwide distribution based on serotyping and sequence typing. Emerging Microbes and Infection Journal 3(1), 1-20.

Jeffrey, J. Z., Locke, A. K., Alejandro, R., \& Kent, J. S. (2012). Diseases of Swine. Iowa, USA: WileyBlackwell.

Jong, A. D., Simjee, S., Garch, F. E., Moyaert, H., Rose, M., Youala, M., \& Dry, M. (2018). Antimicrobial susceptibility of enterococci recovered from healthy cattle, pigs and chickens in nine EU countries (EASSA Study) to critically important antibiotics. Veterinary Microbiology 216, 168-175.

Laxminarayan, R. (2013). Antibiotic resistance-the need for global solutions. Lancet Infectious Diseases 13(12), 1057-1098.

Nagai, S., Someno, S., \& Yagihashi, T. (1994). Differentiation of toxigenic from nontoxigenic isolates of Pasteurella multocida by PCR. Journal of Clinical Microbiology 32(4), 1004-1010.

Nedbalcova, K., Satran, P., Jaglic, Z., Ondriasova, R., \& Kucerova, Z. J. V. M. (2006). Haemophilus parasuis and Glässer's disease in pigs: a review. Veterinarni Medicina 51(5), 168-179. 
Xue, Y., Zhao, Z. Q., Chen, Y., Zou, H. Y., \& He, Q. (2009). Development of a rapid multiplex PCR for stimultaneous detection of five major pathogenic bacteria of respiratory tract disease in swine. Journal of Animal Husbandry and Veterinary Medicine 40, 1222-1228.

Zhao, Z., Xue, Y., Wang, C., Ding, K., Wu, B., He, Q., Cheng, X., \& Chen, H. (2011). Antimicrobial susceptibility of Bordetella bronchiseptica isolates from pigs with respiratory diseases on farms in China. Journal of Veterinary Medical Science 73(1), 103-106.
Zhang, Q. Q., Ying, G. G., Pan, C. G., Liu, Y. S., \& Zhao, J. L. (2015). Comprehensive evaluation of antibiotics emission and fate in the river basins of China: source analysis, multimedia modeling, and linkage to bacterial resistance. Environmental Science Technology 49, 6772-6782. 